\title{
Catabolism of L-rhamnose in A. nidulans proceeds via the non-phosphorylated pathway and is glucose repressed by a CreA-independent mechanism
}

\author{
Andrew P. MacCabe ${ }^{1}$, Elpinickie I. Ninou ${ }^{1,2}$, Ester Pardo $^{1,3}$ and Margarita Orejas ${ }^{1 *}(0)$
}

\begin{abstract}
L-rhamnose (6-deoxy-mannose) occurs in nature mainly as a component of certain plant structural polysaccharides and bioactive metabolites but has also been found in some microorganisms and animals. The release of L-rhamnose from these substrates is catalysed by extracellular enzymes including a-L-rhamnosidases, the production of which is induced in its presence. The free sugar enters cells via specific uptake systems where it can be metabolized. Of two L-rhamnose catabolic pathways currently known in microorganisms a non-phosphorylated pathway has been identified in fungi and some bacteria but little is known of the regulatory mechanisms governing it in fungi. In this study two genes (IraA and IraB) are predicted to be involved in the catabolism of L-rhamnose, along with IraC, in the filamentous fungus Aspergillus nidulans. Transcription of all three is co-regulated with that of the genes encoding a-L-rhamnosidases, i.e. induction mediated by the L-rhamnose-responsive transcription factor RhaR and repression of induction in the presence of glucose via a CreA-independent mechanism. The participation of IraA/AN4186 (encoding L-rhamnose dehydrogenase) in L-rhamnose catabolism was revealed by the phenotypes of knock-out mutants and their complemented strains. IraA deletion negatively affects both growth on L-rhamnose and the synthesis of a-L-rhamnosidases, indicating not only the indispensability of this pathway for L-rhamnose utilization but also that a metabolite derived from this sugar is the true physiological inducer.
\end{abstract}

Keywords: Aspergillus nidulans, L-rhamnose catabolism, Transcriptional regulation, RhaR, CCR, CreA-independent, LRA, IraA/AN4186, L-rhamnose dehydrogenase, RT-qPCR, a-L-rhamnosidases

\section{Introduction}

Metabolic versatility enables microorganisms to deal with fluctuating abiotic conditions and heterogeneous biological interactions, conferring the advantageous ability to utilise a diverse range of natural substrates as nutrients. To be able to metabolize complex organic

*Correspondence: morejas@iata.csic.es

${ }^{1}$ Instituto de Agroquímica y Tecnología de Alimentos (IATA), Consejo Superior de Investigaciones Científicas (CSIC), c/Catedrático Agustín

Escardino Benlloch 7, 46980 Paterna, Valencia, Spain

Full list of author information is available at the end of the article polymers microorganisms must produce a variety of extracellular enzymes that catalyse polymer deconstruction into subunits that can be imported into the cell by transporter proteins. The subsequent catabolism of these soluble nutrients (e.g. sugars) occurs via metabolic pathways encoded by genes that may or may not be organised in clusters. Expression of an appropriate catabolic pathway can be critical for survival and microorganisms have developed sophisticated regulatory mechanisms to ensure the preferential use of those compounds that are most easily catabolized such as glucose; the use of alternative pathways is restricted to those conditions under otherwise in a credit line to the material. If material is not included in the article's Creative Commons licence and your intended use is not permitted by statutory regulation or exceeds the permitted use, you will need to obtain permission directly from the copyright holder. To view a copy of this licence, visit http://creativecommons.org/licenses/by/4.0/. The Creative Commons Public Domain Dedication waiver (http://creativecommons.org/publicdomain/zero/1.0/) applies to the data made available in this article, unless otherwise stated in a credit line to the data. 
which they are absolutely required (e.g. in the presence of a secondary carbon source when a preferred carbon source is not available).

Classified as a rare sugar due to its far lower abundance in nature compared to others, L-rhamnose (6-deoxyL-mannose) occurs mainly as a component of certain plant structural polysaccharides though it has also been found in some microorganisms [1] and rarely in animal tissues [2]. In plants it is widely distributed in the primary cell wall pectic polysaccharides rhamnogalacturonan (RG) I and RGII (reviewed in [3-5]), in the seaweed polysaccharide ulvan (reviewed in [6,7]), as well as in hemicelluloses, glycoproteins and diverse secondary metabolites (e.g. anthocyanins, flavonoids and terpenoids) ([8] and references therein). L-rhamnose can be used by many microbes as a nutrient and also acts as a signalling molecule provoking changes in gene expression. In this regard a number of microorganisms including certain yeasts and filamentous fungi have evolved L-rhamnose-inducible uptake and enzymatic systems that permit the utilization of this sugar as a sole carbon source when preferred carbon sources such as glucose are absent [9-13].

Two pathways have been described for the microbial assimilation of L-rhamnose (Additional file 1: Figure S1A). In many bacterial species such as Escherichia coli L-rhamnose is catabolized to dihydroxyacetone phosphate (DHAP) and L-lactaldehyde via the canonical phosphorylated pathway involving the products of the three genes rhaA (L-rhamnose isomerase), rhaB (L-rhamnulose kinase) and rhaC (rhamnulose-1-P aldolase) $([9,14,15]$ and references therein). An alternative catabolic pathway involving non-phosphorylated intermediates has also been described in the Dothideomycete yeast-like fungus Aureobasidium (aka Pullularia) pullulans and the Saccharomycete yeasts Scheffersomyces (aka Pichia) stipitis and Debaryomyces polymorphus [16-18]. In the latter oxidative route L-rhamnose is enzymatically converted into pyruvate and lactaldehyde by four consecutive reactions catalysed by L-rhamnose-1-dehydrogenase (LRA1), L-rhamnono- $\gamma$-lactonase (LRA2), L-rhamnonate dehydratase (LRA3) and L-2-keto-3-deoxyrhamnonate (L-KDR) aldolase (LRA4). The four enzymes of S. stipitis have been shown to be encoded in a gene cluster named LRA (Additional file 1: Figure S1B) and conservation or partial conservation of this cluster is apparent in other L-rhamnose utilizing yeasts (e.g. Candida lusitaniae), in various filamentous fungi (e.g. Fusarium graminearum, Aspergillus fumigatus and Cryptococcus neoformans) and in some bacteria such as Azotobacter vinelandii and Burkholderia cenocepacia [10,11]. Modified versions of these two catabolic pathways have also been identified in bacteria $[9,19]$. Whilst LRA1-LRA4 in S. stipitis, LRA4 in
Pichia pastoris and the first three catabolic genes $\operatorname{lraA}$, lraB and lraC (clustered) of Aspergillus niger have been functionally characterized [10, 11, 20-22], knowledge of the regulatory mechanisms controlling L-rhamnose catabolism in fungi is sparse, especially carbon catabolite repression (CCR) induced by glucose. In A. pullulans and S. stipitis it has been reported that L-rhamnose-1-dehydrogenase activity is induced by $\mathrm{L}$-rhamnose and that this induction is repressed by D-glucose $[17,23,24]$ but the corresponding transcription factors are unknown.

Similarly to other fungi, $A$. nidulans is able to grow on L-rhamnose as a sole carbon source [25]. Homology searches have identified genomically scattered orthologous sequences potentially encoding the first three steps of the non-phosphorylated catabolic pathway (the current work; Additional file 1: Figure S1B) but to date none has been functionally characterized in this fungus. Thus their involvement in the assimilation of this sugar and the function of the non-phosphorylated pathway in A. nidulans have yet to be formally demonstrated. We have previously shown that deletion of the regulatory gene rhaR (encodes the $\mathrm{Zn}_{2} \mathrm{Cys}_{6} \mathrm{~L}$-rhamnose-responsive transcription activator RhaR) in both A. nidulans and Neurospora crassa (AN5673 and NCU09033, respectively) negatively affects the ability of these fungi to grow on L-rhamnose as a sole carbon source, and in $A$. nidulans this may be contributed at least in part through its influence on the expression of $\operatorname{lraC}$ [26]. Although rhaR is divergently transcribed from the adjacent lraC gene in $A$. nidulans (and other filamentous fungi; Additional file 1: Figure $\mathrm{S1B}$ ), rhaR and $\operatorname{lra} C$ are not co-regulated: whereas expression of rhaR is constitutive that of $\operatorname{lraC}$ is induced when L-rhamnose is present as sole carbon source and this is mediated by RhaR [26]. In the presence of L-rhamnose RhaR also induces the expression of the $A$. nidulans genes rhaA and rhaE (encoding two GH78 $\alpha-\mathrm{L}-$ rhamnosidases that provide the inducing carbon source) which are thus co-expressed with $\operatorname{lraC}$ [26]. Whether other lra genes are subject to induction by RhaR in the presence of L-rhamnose is not known. Nor is it known whether the L-rhamnose catabolic pathway is repressed by glucose and if so what role if any is played by the wide domain repressor CreA, the only carbon catabolite repressor protein known to date in filamentous fungi. In regard to the later, we have previously shown that, unlike many other glycoside hydrolase genes, rhaA and $r h a E$ are repressed by glucose and other carbon sources in a manner independent of CreA. In addition, studies on mixed carbon sources indicated that inducer exclusion may play a prominent role in $\alpha-\mathrm{L}$-rhamnosidase gene regulation [12].

The characterization of catabolic genes and especially knowledge of their regulation can provide a conceptual 
framework upon which strategies can be designed to adjust the flux of inducers and repressors into the fungal cell in order to modulate the productivity of fungal cell factories. The present study examines the roles played by L-rhamnose and $\mathrm{D}$-glucose in controlling processes related to the metabolism of L-rhamnose in A. nidulans. Specifically, we addressed the following points: (1) to establish whether $A$. nidulans catabolizes L-rhamnose via the non-phosphorylated/oxidative LRA pathway, (2) to characterize a gene from this pathway, demonstrating its function and determining whether L-rhamnose catabolism occurs exclusively via this route, (3) to determine whether L-rhamnose or a product of its catabolism is the intracellular signalling molecule that induces gene expression, and (4) to establish whether glucose represses the lra genes and the role played by CreA if any.

\section{Materials and methods}

\section{Strains, media and growth conditions}

Escherichia coli strain DH5 $\alpha$ (supE44, $\Delta \mathrm{U} 169$ ( $\phi 80$ lacZ $\Delta \mathrm{M} 15$, hsdR17, recA1, endA1, gyrA96, thi1, relA1)) was used as the host for cloning experiments and plasmid amplification. Fungal strains used in this study are listed in Table 1. Non-homologous end-joining (NHEJ)-deficient $A$. nidulans strain AR198 (argB2; pyroA4, $\triangle n k u A:: \operatorname{argB}$; riboB2; AKA TN02A21, [27]) was used to delete lraA/AN4186. AR271 (argB2; pyroA4, $\triangle n k u A:: \arg B$; riboB2::Af_riboB; [26]) was used as a riboflavin prototrophic (nutritional) control strain isogenic to gene deletion mutants generated in AR198. A. nidulans wild type AR5 (biA1) and AR305 (creA ${ }^{d} 30$, biA1) strains were used for expression analyses.
E. coli was grown in Luria-Bertani (LB) medium (1\% $\mathrm{w} / \mathrm{v}$ tryptone, $0.5 \% \mathrm{w} / \mathrm{v}$ yeast extract, $1 \% \mathrm{w} / \mathrm{v} \mathrm{NaCl}$ ) with $100 \mu \mathrm{g} / \mathrm{ml}$ ampicillin when selection was required. $A$. nidulans strains were grown in either minimal (MM), $\mathrm{MM}+0.5 \% \mathrm{w} / \mathrm{v}$ yeast extract (YMM) or complete (CM) media $[28,29]$ containing $1 \% \mathrm{w} / \mathrm{v}$ carbon source, unless specified otherwise, and supplemented with the appropriate requirements. Carbon sources were added from filter-sterilised concentrated stocks. Ammonium tartrate ( $5 \mathrm{mM})$ was used as the nitrogen source unless stated otherwise. For solid media $1.5 \% \mathrm{w} / \mathrm{v}$ agar was added.

For the evaluation of $\alpha$-L-rhamnosidase and L-rhamnose dehydrogenase activities in transfer experiments, mycelial biomass was generated from an inoculation yielding a final titre of $5 \times 10^{6}$ spores $/ \mathrm{ml}$ in YMM to which $2 \%$ lactose was added as the sole carbon source. Growth was conducted for $18 \mathrm{~h}$ at $37{ }^{\circ} \mathrm{C}$ with orbital shaking at 180-200 rpm in Erlenmeyer flasks respecting a 5:1 ratio between the total flask volume and the volume of the liquid culture. Mycelia were harvested, washed with MM lacking a carbon source and transferred $(\sim 1 \mathrm{~g}$ drained wet weight) to $100 \mathrm{ml}$ Erlenmeyer flasks containing $20 \mathrm{ml}$ of induction medium (YMM $+1 \% \mathrm{~L}$-rhamnose as the sole carbon source). Lactose was chosen for initial growth of mycelia since previous studies in our group established it to be the least repressive carbon source regarding rhamnosidase synthesis and rhamnosidase gene induction in $A$. nidulans [12, 25].

For RNA extraction in transfer experiments, mycelial biomass was generated from spores inoculated $\left(5 \times 10^{6}\right.$ spores $/ \mathrm{ml}$ ) into YMM $+5 \mathrm{mM}$ urea (nitrogen source) to which $0.1 \%$ fructose was added as the sole carbon source. After $18 \mathrm{~h}$ of growth as described above, mycelia were

Table 1 Aspergillus nidulans strains used in this study

\begin{tabular}{|c|c|c|c|}
\hline Strain code & Name & Genotype $^{a}$ & References \\
\hline AR5 & biA1 & biA1 & M. A. Peñalva (CIB/CSIC) \\
\hline AR305 & $\operatorname{creA}^{d} 30$ & biA1, $\operatorname{creA}^{d} 30$ & M. A. Peñalva/ E. Espeso (CIB/CSIC) \\
\hline AR198 & $\mathrm{TN} 02 \mathrm{~A} 21$ & $\triangle n k u A:: \arg B, \arg B 2$, riboB2, pyroA4 & {$[27]$} \\
\hline AR225 & $\triangle r h a R$ & $\triangle n k u A:: \arg B, \arg B 2$, riboB2, $\triangle$ rhaR::Af_riboB, pyroA4 & {$[26]$} \\
\hline AR271 & & $\triangle n k u A:: \arg B, \arg B 2$, riboB2::Af_riboB, pyroA4 & {$[26]$} \\
\hline AR274 & FGSC A4 & & {$[28]$} \\
\hline AR245 & & $\triangle n k u A:: \arg B, \arg B 2, \triangle \operatorname{raA}: . A f \_r i b o B$, riboB2, pyroA4 & This work \\
\hline AR246 & & $\triangle n k u A:: \arg B, \arg B 2, \triangle \operatorname{lra} A: . A f \_r i b o B$, riboB2, pyroA4 & This work \\
\hline AR247 & & $\triangle n k u A:: \arg B, \arg B 2, \triangle \operatorname{lra} A: . A f \_r i b o B$, riboB2, pyroA4 & This work \\
\hline AR248 & & $\triangle n k u A:: \arg B, \arg B 2, \triangle \operatorname{raA}: . A f \_r i b o B$, riboB2, pyroA4 & This work \\
\hline AR501 & & $\triangle n k u A:: \arg B, \arg B 2, \triangle \operatorname{raA}: . A f \_r i b o B$, riboB2, $\triangle p y r o A 4: . /$ raA-Af_pyroA & This work \\
\hline AR502 & & $\triangle n k u A:: \arg B, \arg B 2, \triangle$ lraA::Af_riboB, riboB2, $\triangle p y r o A 4: . /$ raA-Af_pyroA & This work \\
\hline AR503 & & $\triangle n k u A:: \arg B, \arg B 2, \triangle$ raA $: . A f \_r i b o B$, riboB2, $\triangle p y r o A 4: . /$ raA-Af_pyroA & This work \\
\hline AR504 & & $\triangle n k u A:: \arg B, \arg B 2, \triangle \operatorname{raA}:$ Af_riboB, riboB2, $\triangle$ pyroA4:.IraA-Af_pyroA & This work \\
\hline
\end{tabular}

${ }^{a}$ With the exception of AR274 all the A. nidulans strains carry the mutant allele veA1 
harvested, washed with MM lacking a carbon source and transferred $(\sim 1.5 \mathrm{~g})$ to induction medium $(\mathrm{MM}+$ urea; $15 \mathrm{ml}$ ) for $3 \mathrm{~h}$ in which fructose was substituted by L-rhamnose at $1 \%$; inducing-repressing medium substituted fructose with $1 \%$ L-rhamnose $+1 \%$ glucose.

\section{Transformation}

A. nidulans transformation of the NHEJ-deficient $(\triangle n k u A)$ strain AR198 was undertaken based on the methods of Tilburn et al. [30] and Szewczyk et al. [31]. Protoplasts were generated using Vinoflow FCE lysing enzyme (Novozymes) or Lysing Enzymes from Trichoderma harzianum (Sigma).

\section{Nucleic acid procedures}

Standard molecular techniques were as described in Sambrook and Russell [32]. PCR reactions were performed using Phusion High-Fidelity (Thermo Fisher) or Top-Taq (BIORON) DNA polymerases. Restriction and modifying enzymes were sourced from Roche Diagnostics, New England BioLabs and Thermo Fisher. All products were used as recommended by the manufacturer. DNA sequencing was carried out using the BigDye Terminator v3.1 Cycle Sequencing Kit and run on an ABI PRISM 310 Genetic Analyser (Applied Biosystems, USA) in the Central Service for Experimental Research (SCSIE) of the University of Valencia. Chromatograms were analysed using the programs Chromas 2.6.5 or SnapGene Viewer 4.1.9. Genomic DNA extracted from strains AR198 and FGSC A4 (named AR274 in our collection) were used as templates for the isolation of $A$. nidulans fragments by high-fidelity PCR. Oligonucleotide primers used in this study are listed in Additional file 2: Table S1.

For the preparation of total RNA from transfer cultures, mycelial mass was recovered by filtration through Nytal mesh, rapidly pressed dry between sheets of absorbent paper and flash-frozen in liquid nitrogen; if not used immediately the pressed mycelium was stored at $-80{ }^{\circ} \mathrm{C}$. Approximately $200 \mathrm{mg}$ of frozen mycelium was placed in a $2 \mathrm{ml}$ screw-capped tube containing $850 \mu \mathrm{l}$ RNA Plus (MP Biomedicals, USA) and 5 stainless steel homogenisation beads of $2.3 \mathrm{~mm}$ diameter (BioSpec, USA) and homogenised immediately in a mini-Beadbeater (BioSpec) for $10 \mathrm{~s}$ at $4200 \mathrm{rpm}$. Homogenisation was repeated three times more with cooling intervals on ice. The protocol provided with RNA Plus was followed and the resulting isopropanol-pelleted material was washed with $70 \%$ ethanol and further purified as follows: the pellet was dissolved in $500 \mu \mathrm{L}$ DEPC-treated milliQ water, mixed with three volumes of $4 \mathrm{M}$ sodium acetate $(\mathrm{pH} 6)$ and stored at $-20{ }^{\circ} \mathrm{C}$ for $1-2 \mathrm{~h}$; RNA was recovered by centrifugation $\left(12,000 \mathrm{~g}\right.$ for $20 \mathrm{~min}$ at $\left.4{ }^{\circ} \mathrm{C}\right)$, dissolved in DEPC-treated milliQ water and re-precipitated with sodium acetate and ethanol; after recovery by centrifugation and washing with $70 \%$ ethanol the pellet was completely dissolved in $750 \mu \mathrm{l}$ DEPC-treated milliQ water and $250 \mu \mathrm{l}$ of $10 \mathrm{M}$ lithium chloride solution was then added; after mixing, the sample was incubated for at least $1 \mathrm{~h}$ at $-20{ }^{\circ} \mathrm{C}$ and RNA was subsequently recovered by centrifugation; the final pellet was again dissolved in DEPC-treated milliQ water, re-precipitated with sodium acetate and ethanol, washed with $70 \%$ ethanol and finally dissolved in $100 \mu \mathrm{l}$ DEPC-treated milliQ water. RNA concentration and purity were assessed using a NanoDrop ND-1000 Spectrophotometer (Nano-Drop Technologies) and RNA integrity was verified by agarose gel electrophoresis.

\section{RNA sequencing (RNA-Seq)}

cDNA library construction (TruSeq Stranded mRNA kit-Illumina) and sequencing (single-end $1 \times 75 \mathrm{bp}$; Illumina Nextseq 550) were performed by the Central Service for Experimental Research (SCSIE) of the University of Valencia using total RNA as starting material. Prior to its use, the purity and integrity of the total RNA was assessed using a 2100 Bioanalyser (Agilent) and quantified by Qubit fluorometry (ThermoFisher). The TruSeq kit was used following the manufacturer's instructions. Read quality was checked using FastQC v0.11.3 (https://www.bioinformatics.babraham.ac.uk/ projects/fastqc/) both before and after pre-processing. Raw data were pre-processed with Cutadapt v1.8.3 [33] to remove adapter sequences, filter reads of $<20 \mathrm{nt}$ and trim $3^{\prime}$ ends using a base quality threshold of 28 . Reads were mapped to the $A$. nidulans genome (AspGD version s10-m04-r16) using Tophat v2.1.0 [34]. Raw data quantification was done with Seqmonk v1.42 (https:// www.bioinformatics.babraham.ac.uk/projects/seqmo $\mathrm{nk} /$ ) and expressed as reads per million mapped reads (RPM). Within the SARTools R package [35], DESeq2 v1.18.1 [36] was used for differential expression analysis of raw counts; the false discovery rate (FDR) was obtained from $p$-values using the Benjamini-Hochberg procedure. Only $p$-values below 0.05 were used to identify significant differences in gene expression.

\section{Semi-quantitative reverse-transcriptase PCR (RT-sqPCR) and quantitative PCR (RT-qPCR)}

To prepare template material for RT-qPCR, $20 \mu \mathrm{g}$ of total RNA was treated with $1 \mu \mathrm{l}(10 \mathrm{U})$ RNase-free DNaseI (Roche) in the presence of $0.5 \mu \mathrm{l}(20 \mathrm{U}) \mathrm{RNa}$ seOUT (Invitrogen) in a final volume of $50 \mu \mathrm{l}$ at $37^{\circ} \mathrm{C}$ for $30 \mathrm{~min}$. DNase activity was inactivated at $75^{\circ} \mathrm{C}$ for $10 \mathrm{~min}$ followed by snap chilling on ice for $5 \mathrm{~min}$. After a very brief spin, $5 \mu \mathrm{l}$ of this preparation was added to a PCR tube along with $6 \mu \mathrm{DEPC}$-treated milliQ water, $1 \mu \mathrm{l}$ of oligo $(\mathrm{dT})_{12-18}$ primer $(0.5 \mu \mathrm{g} / \mu \mathrm{l})$ and 
$1 \mu \mathrm{l}$ of dNTPs (10 mM each). This mixture was incubated at $65{ }^{\circ} \mathrm{C}$ for $5 \mathrm{~min}$ and then snap chilled on ice. After a very brief spin, the following components were added and mixed and the whole was incubated for $1 \mathrm{~h}$ at $50{ }^{\circ} \mathrm{C}: 4 \mu \mathrm{l} 4 \times$ first-strand buffer (Invitrogen), $1 \mu \mathrm{l}$ DTT $(0.1 \mathrm{M}), 1 \mu \mathrm{l}(40 \mathrm{U})$ RNaseOUT and $1 \mu \mathrm{l}(200 \mathrm{U})$ Superscript III reverse transcriptase (Invitrogen). Superscript was inactivated by incubation at $70{ }^{\circ} \mathrm{C}$ for $15 \mathrm{~min}$. Amplification of cDNA targets was performed using an aliquot ( $2 \mu \mathrm{l} ; 1 / 10$ th) of the $\mathrm{RT}$ reaction and specific primers in final volumes of $10 \mu \mathrm{l}$.

RT-sqPCR: sense and antisense oligonucleotide primers were located either side of intron/exon boundaries to differentiate genomic and cDNA sequences. The actin gene actA (AN6542) was used as a loading control. To confirm that PCR amplifications were in a linear range, cycle titrations were performed for each gene as described previously [26]. From these analyses, 24 cycles were determined to be optimal for actA (primers 97 and 98) and 25 cycles for $\operatorname{lraA} / \mathrm{AN} 4186$ (primers 120 and 121) in the AR5 (biA1) wild type strain. The data were confirmed in two independent experiments.

RT-qPCR: reactions were performed using SYBR Green PCR reagents (Roche) in a LightCycler 480 instrument (Roche). Three biological replicates were undertaken for each biological condition and qPCR of each was performed in triplicate (technical replicates)-these procedures were conducted at different times and by more than one operator. Cycling conditions were: $95{ }^{\circ} \mathrm{C}$ for $5 \mathrm{~min}$, followed by 45 amplification cycles of $10 \mathrm{~s}$ at $95{ }^{\circ} \mathrm{C}, 10 \mathrm{~s}$ at $60^{\circ} \mathrm{C}, 12 \mathrm{~s}$ at $72{ }^{\circ} \mathrm{C}$; for product melting data a single cycle of $1 \mathrm{~min}$ at $65^{\circ} \mathrm{C}$ followed by a linear increase in temperature to $95^{\circ} \mathrm{C}$ at a rate of $0.11{ }^{\circ} \mathrm{C} / \mathrm{s}$ completed the run. Cycle threshold (Ct) data were obtained using the LightCycler 480 software package v1.5.0.39. The genes encoding histone H2B/AN3469 (primers 394 and 395) and beta-tubulin benA/AN1182 (primers 386 and 387) were used as references. The stability of expression of these genes in our biological samples was verified using geNorm [37]. Primers for RT-qPCR were designed with the help of the Primer-BLAST tool (NCBI) and were chosen to cross intron/exon junctions where feasible. The specificity of primer pair products was confirmed by gel electrophoresis where possible, and melting curve analysis was carried out in all cases. Primer pair efficiencies where obtained by qPCR (values are given in Additional file 2: Table S1). Relative quantification of reference-gene-normalized target genes was determined using the Relative Expression Software Tool (Multiple Condition Solver REST-MCS v2) [38] and REST 2009 (Qiagen).

\section{Generation of IraA disruption mutants and complemented strains}

In order to generate an A. nidulans AN4186 null mutant a deletion cassette was constructed in pBluescript SK $(+)$ (Stratagene). gDNA sequences flanking the AN4186 CDS were obtained as PCR-generated fragments: oligonucleotide pair LRA1_NotI (61) and LRA1_rev (62), which incorporate NotI and EcoRI restriction sites respectively, were used to amplify the $5^{\prime}$-UTR (1779 bp) region; oligonucleotide pair LRA1_dir (63) and LRA1_KpnI (64), which incorporate EcoRI and NotI-KpnI restriction sites respectively, amplified the $3^{\prime}$-UTR (1514 bp). These PCR fragments were cloned into the NotI and KpnI sites of pBluescript SK $(+)$ yielding plasmid pE406 and the absence of mutations was confirmed by sequencing (oligonucleotides 141 and 142). A $1.9 \mathrm{~kb}$ EcoRI fragment comprising the A. fumigatus riboB (Afu1g13300) expression cassette (Af_riboB, obtained from plasmid pTN2 [27]) was then subcloned into the EcoRI site of pE406 to generate $\mathrm{pE} 407$. Deletion of AN4186 was achieved by transforming protoplasts of strain AR198 with the NotI deletion cassette $(5.3 \mathrm{~kb})$ isolated from pE407 and selecting for transformants able to grow in the absence of riboflavin. Riboflavin prototrophs were purified, tested for growth on L-rhamnose and analysed by diagnostic PCR and Southern blot analysis.

To complement the AN4186 deletion, a DNA fragment comprising the wild type AN4186 locus including sequences upstream and downstream of the CDS (804 and 623 bp respectively) was generated by highfidelity PCR from gDNA of strain AR198 using the oligonucleotide pair 547-548 and cloned into the SmaI site of pVAL159 yielding plasmid pE528 (pVAL159 comprises the A. fumigatus pyroA cassette from pTN1 [27] flanked by the $A$. nidulans pyroA/AN7725 upstream and downstream sequences (UTR) cloned in pBluescript SK $(+))$. A $5.8 \mathrm{~kb}$ fragment obtained by high-fidelity PCR from pE528 using primers 602 and 603 comprising four DNA sequence elements (5'UTR pyroA/AN7725-lraAAf_pyroA-3'UTR pyroA/AN7725) was used to transform $\triangle$ lraA strain AR247 and ectopically express $\operatorname{lraA}$ at the AN7725 locus. Transformants C1-C4 (AR501-AR504) were selected for growth in the absence of pyridoxine, and the integrities of the $\operatorname{lra\mathrm {A}}$ complementing cassette as well as the original $\operatorname{lraA}$ disruption cassette present in AR247 were confirmed by PCR (see "Results and discussion").

\section{a-L-Rhamnosidase assays}

$\alpha$-L-Rhamnosidase activity in cell-free extracts was measured using the artificial substrate $p$-nitrophenyl$\alpha$-L-rhamnopyranoside ( $p$ NPR). The release of $p$-nitrophenol was measured spectrophotometrically 
at $400 \mathrm{~nm}$. The assay was performed in 96-well microtitre plates in final volumes of $250 \mu \mathrm{l}$ using $1.4 \mathrm{mM}$ substrate in $100 \mathrm{mM}$ McIlvaine buffer (citrate-phosphate buffer) $\mathrm{pH} 4.0$ and incubated at $50{ }^{\circ} \mathrm{C}$ for $15 \mathrm{~min}$ with shaking essentially as described previously [39]. To assess the $\alpha-\mathrm{L}$-rhamnosidase activity of $A$. nidulans colonies in vivo, MM plates were supplemented with $40 \mu \mathrm{M}$ 4-methylumbelliferyl $\alpha$-L-rhamnopyranoside (MUR) and $1 \mathrm{mM}$ Mcllvaine buffer $\mathrm{pH} 4.0$ as described [12]. Cell-free extracts were from duplicate growths and rhamnosidase assays were performed in duplicate.

\section{L-Rhamnose dehydrogenase activity}

L-Rhamnose dehydrogenase activity was measured by detecting the formation of NADH as described previously [20] in crude cell-free extracts obtained by sonication (1 pulse of $1 \mathrm{~min}$ and 5 pulses of $30 \mathrm{~s}$, with resting periods in ice) and subsequent centrifugation at $4{ }^{\circ} \mathrm{C}$. Reactions were carried out at $37{ }^{\circ} \mathrm{C}$ for $6 \mathrm{~h}$ in final volumes of $200 \mu \mathrm{l}$ comprising $45 \mu \mathrm{l}$ Tris $-\mathrm{HCl}$ $100 \mathrm{mM}$ (pH 8.0), $50 \mu \mathrm{l}$ Tris-HCl $200 \mathrm{mM}$ (pH 8.0), $10 \mu \mathrm{l}$ distilled water, $40 \mu \mathrm{l}$ NAD $2 \mathrm{mM}, 5 \mu \mathrm{l}$ extract (三10 $\mu \mathrm{g}$ total protein) and $50 \mu \mathrm{l} 50 \mathrm{mM}$ L-rhamnose (the latter was substituted by $50 \mu \mathrm{l}$ Tris- $\mathrm{HCl} 100 \mathrm{mM}$ for the control). A Polarstar Omega microplate reader (BMG LabTech) was used to measure and analyse the data. The increase in absorbance at $340 \mathrm{~nm}$ was used to monitor the formation of NADH. Protein concentrations were measured by the Bradford method using lysozyme as standard [40].

\section{Results and discussion}

In silico evidence for the non-phosphorylated L-rhamnose catabolic pathway in $A$. nidulans

In most bacteria L-rhamnose is converted to L-rhamnulose by L-rhamnose isomerase (EC 5.3.1.14; RhaA [14]) in the first reaction of the canonical phosphorylated pathway. By contrast, in some other bacteria and fungi L-rhamnose is metabolised to L-rhamnono- $\gamma$-lactone by L-rhamnose 1-dehydrogenase (EC 1.1.1.173) which is encoded by the $L R A 1 / R H A 1$ gene (Additional file 1 : Figure S1A) [11, 20]. BlastP searches of the $A$. nidulans genome (AspGD; https://www.aspergillusgenome. org/) failed to identify homologues of the E. coli protein RhaA, strongly suggesting the existence of an alternative pathway for the catabolism of L-rhamnose. Accordingly, a BlastP search using the S. stipitis Lra1/Rha1 protein (jgi|Picst3|50944) as the query sequence identified the protein encoded by locus AN4186 (Table 2) as yielding the best bidirectional hit (271 amino acids (aa) in length; E-value $5 \mathrm{e}-86 ; 63.5 \%$ identity). AN4186 is currently annotated as encoding a putative glucose 1-dehydrogenase $(\mathrm{GudB})$ and is located on chromosome II. The next closest hit was AN1886 (246 aa, E-value 5e-61 and

Table 2 Identification and expression (RPKM) of A. nidulans genes homologous to the LRA genes of S. stipitis

\begin{tabular}{|c|c|c|c|c|c|c|}
\hline Probe (S. stipitis) & $\begin{array}{l}\text { A. nidulans locus } \\
\text { (AspGD) }\end{array}$ & E value $(<1 E-30)$ & $\begin{array}{l}\text { Reverse Blast. Hit } \\
\text { of lowest E value } \\
(<1 \mathrm{e}-30)\end{array}$ & $\begin{array}{l}\text { RPKM: } \\
\text { AR271 } \\
\text { on lactose }\end{array}$ & $\begin{array}{l}\text { RPKM: AR271 } \\
\text { on rhamnose }\end{array}$ & $\begin{array}{l}\text { RPKM: AR225 } \\
\text { on rhamnose }\end{array}$ \\
\hline \multirow{2}{*}{$\begin{array}{l}\text { LRA1/RHA1 } \\
\quad \text { (jgi|Picst3|50944) }\end{array}$} & AN4186 (chr. II) & $5.0 \mathrm{E}-86$ & Picst3|50944 (1.24E-90) & 11 & 983 & 9 \\
\hline & AN1886 (chr. VII) & $5.0 \mathrm{E}-61$ & Picst3|50944 (1.06E-53) & $0.31^{a}$ & $0.49^{a}$ & $0.45^{a}$ \\
\hline LRA2 (jgi|Picst3|63908) & AN3740 (chr. II) & $5.0 \mathrm{E}-56$ & Picst3|63908 (5.18E-42) & 28 & 185 & 35 \\
\hline LRA3 (jgi|Picst3|50672) & AN5672 (chr. V) & $5.0 \mathrm{E}-171$ & Picst3|50672 (0) & 49 & 1785 & 116 \\
\hline \multirow[t]{12}{*}{ LRA4 (jgi|Picst3|64442) } & AN7929 (chr. II) & $9.0 \mathrm{E}-40$ & Picst3:64442 (3.1E-35) & $0^{a}$ & $0.57^{a}$ & 3 \\
\hline & AN0617 (chr. VIII) & $1.0 \mathrm{E}-38$ & Picst3:64442 (2.47E-30) & $0.78^{a}$ & $1.27^{\mathrm{a}}$ & $1.43^{\mathrm{a}}$ \\
\hline & AN10990 (chr. IV) & $1.0 \mathrm{E}-35$ & Picst3:64442 & 6 & 6 & 3 \\
\hline & AN2859 (chr. Vl) & $1.0 \mathrm{E}-33$ & Picst3:64442 & 30 & 28 & 33 \\
\hline & AN1503 (chr. VII) & $2.0 \mathrm{E}-33$ & Picst3:64442 & 55 & 23 & 44 \\
\hline & Rhamnosidases: & & & & & \\
\hline & rhaA/AN10277 & & & $0.55^{\mathrm{a}}$ & 1244 & 3 \\
\hline & rhaE/AN7151 & & & $0.19^{a}$ & 542 & $0.4^{\mathrm{a}}$ \\
\hline & Reference genes: & & & & & \\
\hline & nkuA/AN7753 & & & $1.21^{\mathrm{a}}$ & $1.74^{\mathrm{a}}$ & $1.57^{\mathrm{a}}$ \\
\hline & benA/AN1182 & & & 207 & 246 & 226 \\
\hline & H2B/AN3469 & & & 2490 & 2888 & 2642 \\
\hline
\end{tabular}

Transcript abundances (RPKM, i.e. reads per kilobase of exon model per million mapped reads) are averages of triplicates. A. nidulans strain AR225 is deleted for the transcription activator gene rhaR and AR271 is the corresponding nutritional isogenic rha ${ }^{+}$control

${ }^{a}$ Considered as not expressed 
$55.6 \%$ identity) which is annotated as being a putative tetrahydroxynaphthalene reductase.

RNA-Seq analyses (our work to be published in detail elsewhere) have shown AN4186 transcript abundance (reads per kilobase million-RPKM) to be significantly greater (983 vs 11; DEseq2 $p$-value 1.6E-67) in $A$. nidulans strain AR271 transferred to medium containing L-rhamnose as sole carbon source compared to transfers of the same strain to medium containing lactose, thus indicating specific induction of that locus on L-rhamnose (Table 2). Under inducing conditions (L-rhamnose) AN4186 RPKM were significantly lower in the strain (AR225) deleted for the transcription factor rhaR/AN5673 compared to AR271 (9 vs 983; DEseq2 $p$-value 9.6E-74-Table 2). By contrast, no expression (RKPM) was observed for locus AN1886 under the conditions studied. Taken together these data strongly suggest that AN4186 (named lraA) is the functional homologue of $L R A 1 / R H A 1$. An $\operatorname{lraA}$ cDNA (ATG to STOP) was obtained by high-fidelity PCR using the oligonucleotide pair 120 and 122 and subcloned into the EcoRV site of pBluescript SK (+). The insert (886 bp), present in two independently obtained plasmids pE462 and pE463, was sequenced (oligonucleotides 141 and 142) revealing $\operatorname{lraA}$ to be interrupted by three consensus (GT-AG) introns of 90 , 67 and 50 nucleotides thus confirming its predicted intron/exon structure (AspGD).

In the $S$. stipitis LRA pathway L-rhamnono- $\gamma$-lactone (i.e. the product of the reaction catalysed by Lra1/ Rha1) is further metabolized to L-rhamnonate and subsequently to L-2-keto-3-deoxyrhamnonate (L-KDR) in two consecutive reactions catalysed by L-rhamnono- $\gamma$ lactonase (Lra2; EC 3.1.1.65) and L-rhamnonate dehydratase (Lra3; EC 4.2.1.90) respectively (Additional file 1: Figure S1A). Bidirectional BlastP analyses resulted in the identification of a unique homologue candidate for each enzyme among the repertoire of hypothetical $A$. nidulans proteins: AN3740 (360 aa; E-value 5e-56; 34.7\% identity to Lra2; maps to chromosome II but is located far from AN4186) and AN5672 (425 aa; E-value 5e-171; $66.5 \%$ identity to Lra3; chromosome V). RNA-Seq analyses showed co-expression (i.e. specific induction on L-rhamnose mediated by RhaR) of these two loci with those of AN4186/lraA and the $\alpha$-L-rhamnosidase genes rhaA and rhaE (Table 2). Moreover, as noted previously [26] AN5672 is adjacent to and divergently transcribed from the rhaR (AN5673) regulatory gene, a topology maintained in certain other filamentous ascomycetes (e.g. Neurospora crassa). These data strongly suggest that AN3740 (named lraB) along with AN5672 (named $\operatorname{lraC}$ ) are the functional homologues of $L R A 2$ and $L R A 3$ respectively.
The fourth step in the LRA pathway is the aldolasemediated conversion of L-KDR to pyruvate and L-lactaldehyde. To date, eukaryotic/fungal L-KDR aldolases have only been identified in the related yeasts $S$. stipitis and $P$. pastoris $[11,21]$. Bidirectional BlastP analyses combined with gene expression and gene deletion studies failed to identify the functional homologue of $L R A 4$ in $A$. niger [22]. Our BlastP screening of the $A$. nidulans proteome also failed to reveal a clear $L R A 4$ candidate, yielding instead five low-similarity targets (AN7929 E-value 9e-40, AN0617 1e-38, AN10990 1e-35, AN2859 $1 \mathrm{e}-33$ and AN1503 2e-33). The potential physiological relevance of these loci was assessed by comparing their expression (RPKM) in mycelia transferred to medium containing L-rhamnose with a reference medium containing lactose. Expression of the five loci was either absent or not co-regulated with $\operatorname{lraA}-\operatorname{lraC}$ or the rhamnosidases (Table 2).

From the above, $A$. nidulans genes potentially encoding L-rhamnose catabolic activities have been identified based on sequence homologies with the previously characterized LRA1-LRA3 genes of S. stipitis [11]. RNA-Seq has shown the expression of loci AN4186, AN3740 and AN5672 to coincide with that of the $\alpha$-L-rhamnosidase genes $r h a A$ and $r h a E$, findings that concord with studies in $A$. niger [22, 41] and $N$. crassa [42]. A challenge for our future studies will be the identification of the $A$. nidulans gene encoding the catalytic step corresponding to LRA4. With regard to the latter, AN7929, AN2859 and AN1503 are orthologues of the lraD1, lraD2 and lraD3 loci in $A$. niger, the deletions of each of which failed to affect the ability of $A$. niger to grow on L-rhamnose [22].

\section{Deletion of IraA/AN4186 severely impairs L-rhamnose utilization}

To test whether the predicted LRA pathway is necessary and sufficient for growth on L-rhamnose, targeted deletion of the candidate gene for the first catabolic step (lraA/AN4186) was achieved by replacing its open reading frame (from -17 to -7 relative to the ATG and stop codons respectively) in AR198 with the A. fumigatus ribo $B$ expression cassette (Af_riboB) that can complement the $A$. nidulans riboB2 mutant allele (the mutant allele is deleted for a single cytosine residue (CTT.CGT. CAA... to CTT.gtc.aag...) resulting in a frameshift after Leu285 and protein truncation after the addition of 145 novel amino acids; [26]). The phenotypes of ten clonally purified riboflavin prototrophic transformants together with that of the parental strain AR198 were first assessed on appropriately supplemented MM plates containing either glucose or L-rhamnose as sole carbon source in the presence of either urea (a poor $\mathrm{N}$ source but not a carbon source [43]) or ammonium tartrate (a preferred $\mathrm{N}$ source 

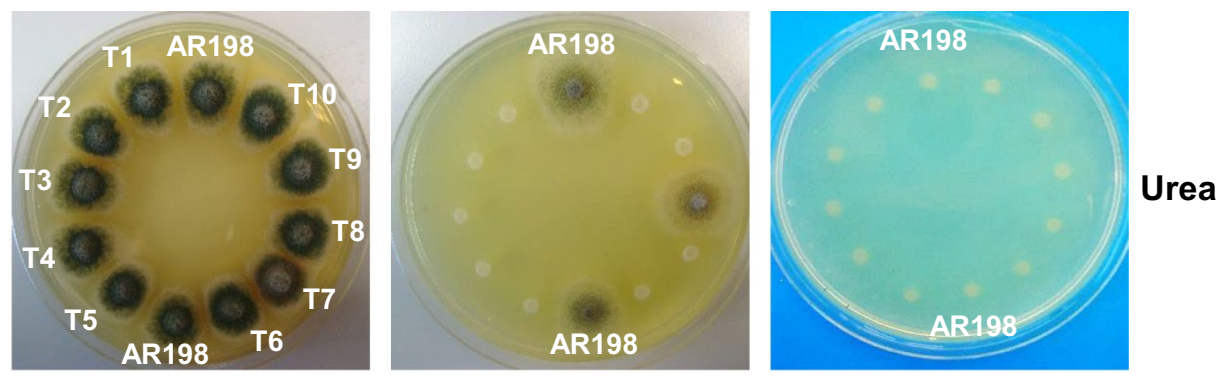

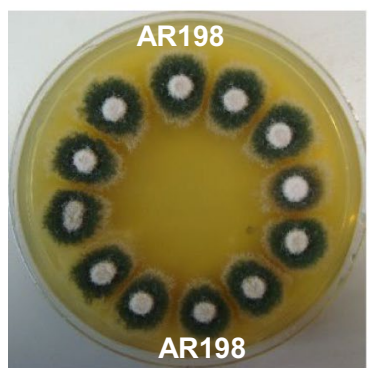

$1 \%$ D-Glucose

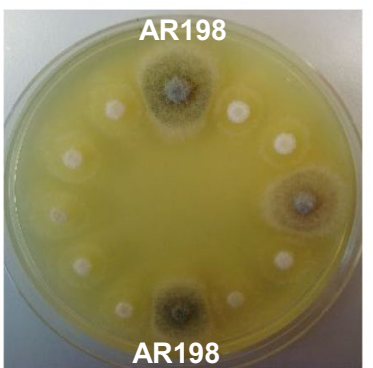

1\% L-Rhamnose

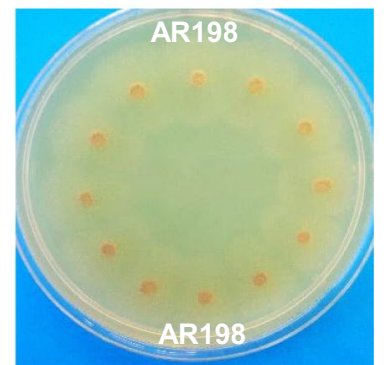

No C source

$\mathrm{NH}_{4}$ Tartrate

Fig. 1 Deletion of IraA/AN4186 leads to reduced growth on L-rhamnose. The untransformed strain (AR198) and riboflavin prototrophic transformants ( $\mathrm{T} 1$ to $\mathrm{T} 10$ ) were grown $\left(3\right.$ days at $37^{\circ} \mathrm{C}$ ) on minimal media supplemented with riboflavin (required by AR198) and pyridoxine (required by all strains) containing different carbon and nitrogen sources. Nine transformants show considerably impaired growth on L-rhamnose. $1.5 \times 10^{5}$ conidia were spotted in $3 \mu$ drops

that can also be used as a poor carbon source). Nine of the transformants showed the same growth as the parental strain on glucose medium. Their growth on L-rhamnose however was severely affected, the mycelial sparsity observed resembling that seen both on control plates lacking sugar (Fig. 1) and rhaR loss-of-function mutants on rhamnose [26]. Similar consequences for rhamnose utilization have also been reported in A. niger [22]. To confirm integration of the disruption cassette at the AN4186 locus, four transformants (T5, T6, T9 and T10, renamed AR245-AR248 respectively) were selected and analysed by diagnostic PCR. Figure $2 \mathrm{a}$ shows oligonucleotide pairs $72-73,72-60$ and $195-73$ yielded fragments of 5.5, 2.0 and $1.8 \mathrm{~kb}$ in all four as predicted for replacement of $l r a A$ by the $A f_{-}$riboB cassette whereas fragments of $4.5 \mathrm{~kb}$ and $2.1 \mathrm{~kb}$ were obtained for the parental strain AR198 with oligo pairs 72-73 and 72-75. Southern blot analysis was consistent with single copy integrations at the homologous locus and the absence of ectopic integrations. Given the identical nature of the phenotype and results of the molecular analyses for the four transformants, AR247 and AR248 were randomly chosen for further work. Growth tests on a range of other carbon sources showed similar growth of these transformants and the control thereby corroborating the specific role of IraA in the utilization of L-rhamnose (Fig. 2b).

\section{Enzymatic analysis of LraA}

To verify the function of the lraA/AN4186 gene product, the $\mathrm{NAD}^{+}$-dependent $\mathrm{L}$-rhamnose-1-dehydrogenase (LraA) activity present in $\Delta$ lraA (AR247 and AR248) and $\mathrm{lraA}^{+}$(AR198) cell-free extracts obtained from cultures transferred to L-rhamnose medium was assayed by direct NADH detection at $340 \mathrm{~nm}$ as described previously [11]. In contrast to AR198, NADH formation was not detected in the deletion mutant extracts upon addition of L-rhamnose (Fig. 3a) thus supporting the identification of AN4186 as the locus that encodes LraA. The data also reveal that under the experimental conditions no other $A$. nidulans enzyme compensates the lack of LraA activity in the $\triangle \mathrm{AN} 4186$ mutants.

Glucose is known to repress L-rhamnose dehydrogenase activity in A.pullulans and S. stipitis [23, 24]. It was therefore of interest to study the kinetics of LraA activity in AR198 cultivated under inducing/repressing conditions ( $1 \% \mathrm{~L}$-rhamnose $+1 \%$ glucose). Figure 3 a shows that the rate of NADH formation was reduced in the presence of glucose hence the effect of glucose on the production of LraA activity in A. nidulans is similar to that observed in the earlier studies.

\section{LraA production is regulated at the level of transcription}

In a first approximation to see whether L-rhamnose induction and carbon catabolite repression (CCR) of 

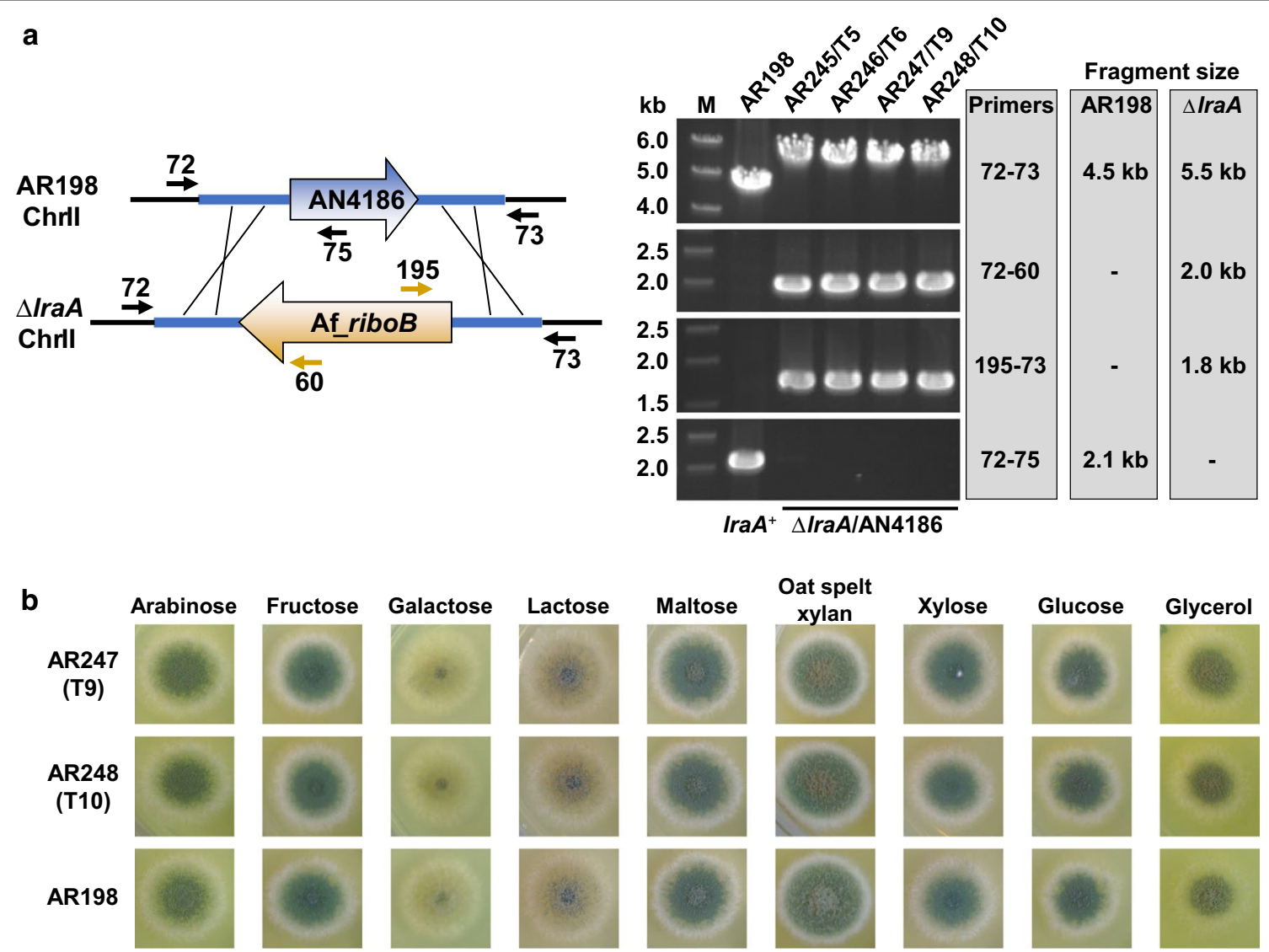

Fig. 2 Identification, gene replacement analysis and growth tests of A. nidulans $\triangle$ rraA mutant strains. a Schematic diagram of the IraA/AN4186 locus in AR198 and the gene replacement event in $\triangle$ IraA strains. Correct replacement of IraA/AN4186 with the Af_riboB expression cassette in four selected transformants (T5, T6, T9 and T10) was verified by the absence or appearance of PCR products of the expected sizes using CDS-located primers $(60,75$ and 195) along with primers 72 and 73 located outside the IraA flanking sequences used in the gene replacement cassette. $\mathbf{b} A$. nidulans $\triangle$ rraA mutant (AR247 and AR248) and untransformed (AR198) strains grown (3 days at $37^{\circ} \mathrm{C}$ ) on solid MM containing different carbon sources (1\%) and supplemented with pyridoxine and riboflavin. Equal numbers of conidia $\left(10^{4}\right)$ were spotted $(2 \mu \mathrm{l})$ from a sterile suspension in 0.005\% Tween 80. Complete genotypes are given in Table 1

the synthesis of LraA in A. nidulans-and by extension the function of the LRA pathway-occurs at the level of transcription, semi-quantitative RT-sqPCR was performed revealing lower levels of $\operatorname{lraA}$ transcript accumulation under inducing/repressing compared to inducing conditions whilst transcript levels of the reference gene actA were similar in both. These data are consistent with glucose repression of LraA production at the level of transcription. That $\operatorname{lraA}$ transcripts were not detected or were much reduced in mycelia transferred to medium either lacking a carbon source or containing a carbon source other than L-rhamnose (Fig. 3b) indicates that transcriptional regulation of IraA occurs by specific induction in the presence of L-rhamnose rather than derepression.

The above genetic and biochemical analyses demonstrate the physiological relevance of the lraA gene in the utilization of L-rhamnose by $A$. nidulans. The inability of knockout strains $(\triangle \operatorname{lraA})$ to grow on L-rhamnose also provides convincing evidence that A. nidulans uses the non-phosphorylated pathway for L-rhamnose catabolism and that there are no alternative routes.

\section{Deletion of IraA impairs the production of a-L-rhamnosidases}

It has been established previously that L-rhamnose induces the expression of the A. nidulans $\alpha-\mathrm{L}-$ rhamnosidase genes at the level of transcription [12] and requires the presence of the $\mathrm{Zn}_{2} \mathrm{Cys}_{6}$ transcription activator RhaR [26]. To investigate whether LraA plays a role in this induction, extracellular $\alpha$-L-rhamnosidase activity was measured in mycelia of $A$. nidulans raA $^{+}$(i.e. AR198 and the nutritional control AR271) and lraA-deleted 

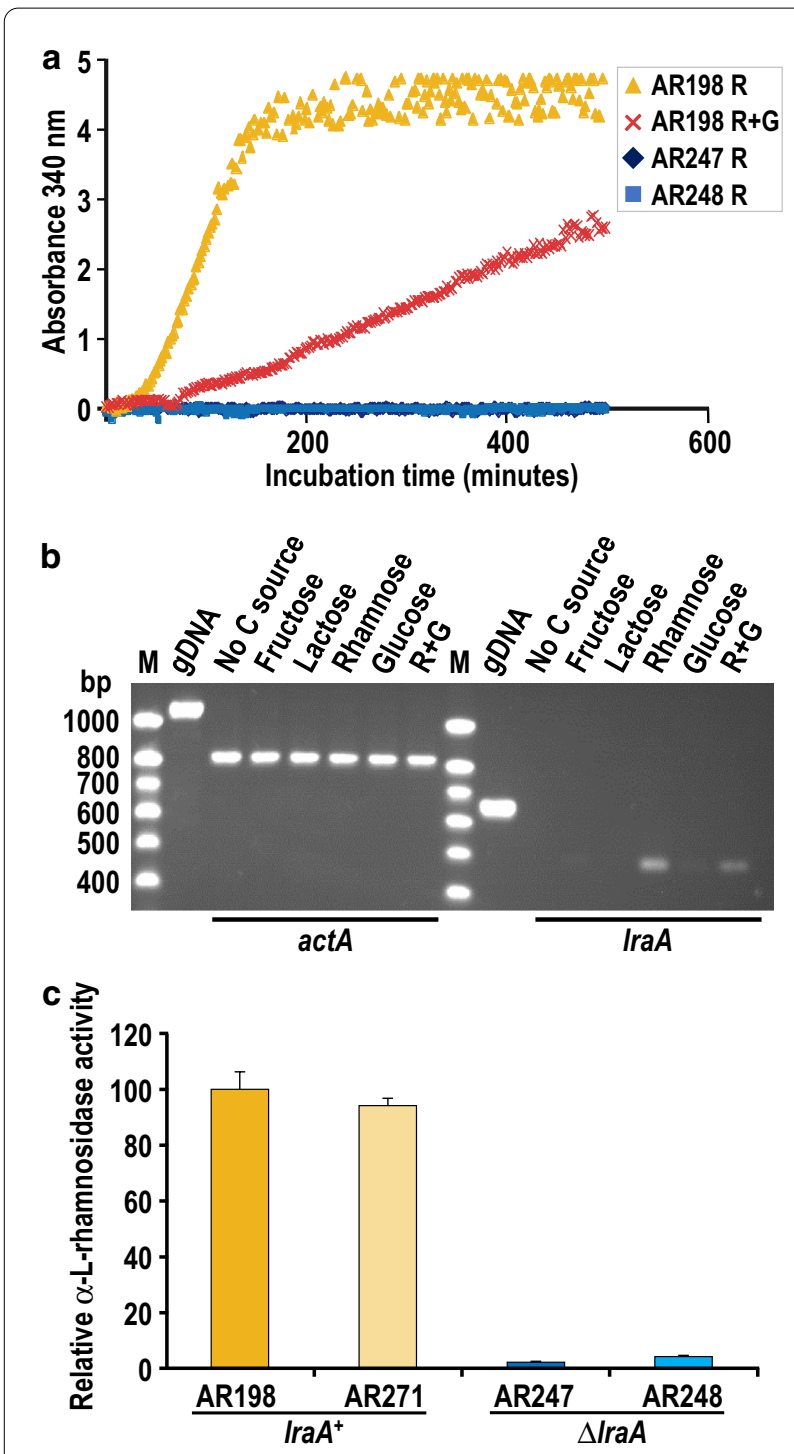

Fig. 3 Physiological function of IraA/AN4186 in L-rhamnose catabolism. a L-Rhamnose dehydrogenase activity: increase in absorbance at $340 \mathrm{~nm}$ upon incubation of L-rhamnose with cell free extracts of untransformed IraA ${ }^{+}$(AR198) and $\triangle$ rraA (AR247 and AR248) strains in the presence of $\mathrm{NAD}^{+}$at $37^{\circ} \mathrm{C}$. $\mathbf{b}$ Expression of /raA in the biA1 wild type control strain AR5 under different growth conditions: RT-sqPCR of RNA isolated from mycelia obtained $3 \mathrm{~h}$ after transfer to media lacking a carbon source (non-inducing/non-repressing conditions), $0.1 \%$ fructose (i.e. the pre-growth conditions), $2 \%$ lactose (non-inducing), 1\% L-rhamnose (inducing), $1 \%$ glucose (repressing), and $1 \%$ L-rhamnose $+1 \%$ glucose ( $+\mathrm{G}$ inducing/repressing). The actin actA gene was used as a constitutive expression control for normalization. Amplifications (PCR) were reduced to 24 (actA) and 25 (IraA) cycles in order to obtain semi-quantitative data. PCR amplifications of gDNA are shown. M, low molecular weight marker (base pairs). c Deletion of IraA/AN4186 dramatically affects the production of a-L-rhamnosidase activity: extracellular a-L-rhamnosidase activities in $/ \mathrm{raA}^{+}$(AR198 and the isogenic nutritional control AR271) and $\triangle \mathrm{r}$ raA (AR247 and AR248) strains $48 \mathrm{~h}$ after transfer to inducing conditions (1\% L-rhamnose). Average activities and standard deviations of duplicates of two independent biological experiments are presented as percentages of those observed in AR198 strains (AR247 and AR248) $48 \mathrm{~h}$ after transfer from 2\% lactose MM to MM containing 1\% L-rhamnose as sole carbon source. In contrast to the $\operatorname{lraA}^{+}$strains $\alpha-\mathrm{L}-$ rhamnosidase activity was drastically reduced in both of the $\triangle l r a A$ mutants $(<5 \%$ of the level in wild type; Fig. 3c). Hence the lraA gene-and therefore the LRA pathway-plays a positive role in the biosynthesis of $\alpha-\mathrm{L}-$ rhamnosidases (enzymes that liberate L-rhamnose from complex substrates).

These data indicate that the physiological inducer of $\alpha$-L-rhamnosidase production in A. nidulans is in fact a product of the L-rhamnose catabolic pathway, thus making this route not only essential for the assimilation of this sugar but also the source of the inducer and hence having a role in regulating transcription.

\section{Re-introduction of IraA into the $\Delta / r a A$ mutant restores the ability to grow on L-rhamnose and produce a-L-rhamnosidases}

To confirm that the $\triangle l r a A$ mutant phenotype is specifically the result of $\operatorname{lraA}$ deletion, ectopic complementation at the pyroA (AN7725) locus was undertaken. An lraA complementation cassette (5'UTR pyroA/ AN7725-lraA expression cassette-Af_pyroA-3'UTR pyroA/AN7725) generated by high-fidelity PCR from plasmid pE528 employing primers 602 and 603 (see "Materials and methods" for details) was used to transform AR247 (relevant genotype $\triangle$ lraA::Af_riboB, $\triangle n k u A:: \arg B$, pyroA4) and four pyridoxine prototrophic transformants (AR501-AR504) were selected and purified. The phenotypes of the latter were tested on solid medium containing L-rhamnose with and without the presence of the fluorogenic artificial substrate MUR: all four were able to grow on L-rhamnose (Fig. 4a) and also degrade MUR (Fig. 4b). As expected, the control strain AR271 (not deleted for $\operatorname{lraA}$ ) was also able to grow on L-rhamnose and hydrolyse MUR whereas the $\triangle$ lraA mutants AR247 and AR248 were not. The $\operatorname{lraA}$ gene is thus able to restore the wild type phenotype as manifested by recovery of the ability to utilise L-rhamnose as a carbon source as well as the production of UV fluorescent halos resulting from MUR degradation by $\alpha$-L-rhamnosidase activity. Diagnostic PCRs (Fig. 4c) confirmed the unaltered nature of the $\triangle$ lraA::Af_riboB deletion on chromosome II and ectopic insertion of the complementing cassette at the pyroA locus (AN7725 on chromosome IV) in all four selected transformants.

These results along with the previous data confirm the role of the $A$. nidulans $\operatorname{lraA}$ gene in the metabolism of $\mathrm{L}$-rhamnose and the induction of $\alpha$-L-rhamnosidase synthesis. 


\section{AR247}

(argB2, $\triangle n k u A:: a r g B$, riboB2, $\Delta$ IraA/AN4186::Af_riboB, pyroA4)

Transforming PCR fragment amplified from $\mathrm{pE} 528$ :

5'UTR pyroA/AN7725 - IraA cassette - Af_pyroA - 3'UTR pyroA/AN7725)

$\downarrow$

AR501-AR504 (C1-C4)

(argB2, $\triangle n k u A:: a r g B$, riboB2, $\Delta /$ raA/AN4186::Af_riboB, $\triangle$ pyroA4::IraA-Af_pyroA)

a

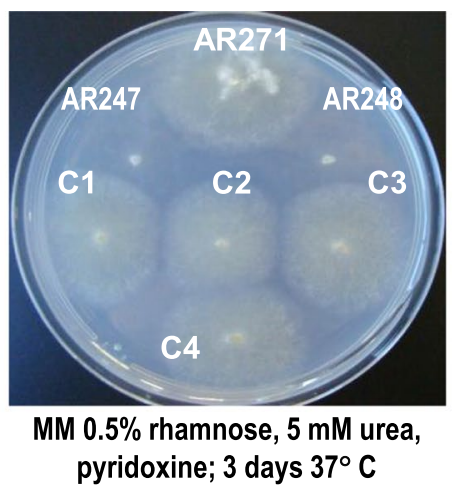

C

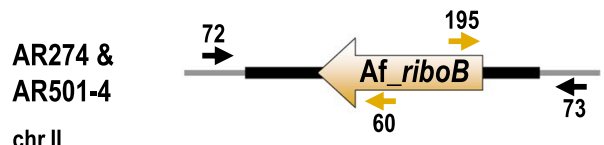

locus AN4186

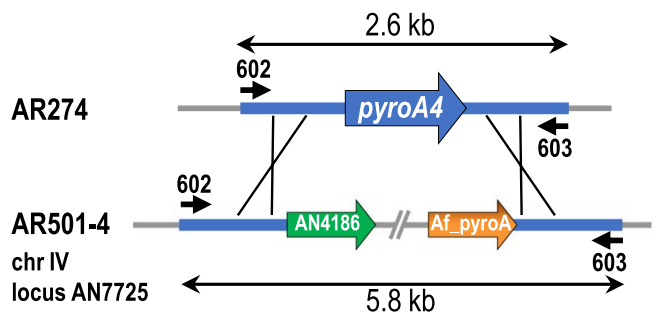

b

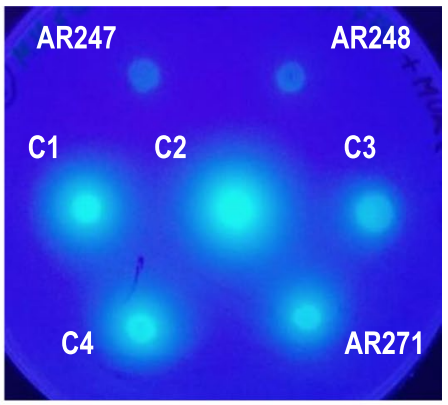

MM $0.5 \%$ rhamnose, $5 \mathrm{mM}$ urea, pyridoxine + MUR; 40 hours RT
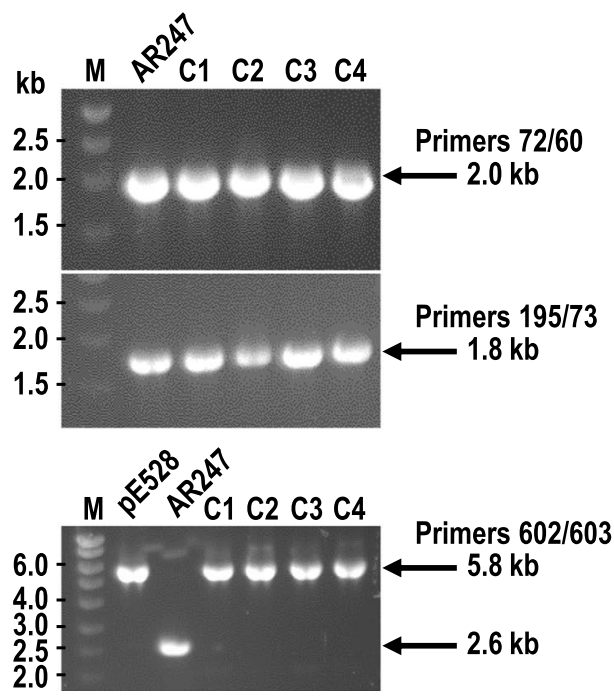

Fig. 4 Ectopic expression of IraA in the $\triangle$ IraA mutant background restores both growth on L-rhamnose and a-L-rhamnosidase activity. Transformation-mediated ectopic replacement of pyroA4 by functional IraA in AR247 ( $\triangle$ rraA). a Plate growth tests of the isogenic Ira $A^{+}$nutritional control AR271, $\triangle$ rraA strains (AR247 and AR248) and four IraA $A^{+}$complemented transformants (C1-C4) on L-rhamnose after 3 days at $37^{\circ} \mathrm{C}$. b MUR-mediated detection (under UV illumination) of a-L-rhamnosidase activity halos produced by IraA-complemented transformants (C1-C4) and control AR271 $\left(\mathrm{IraA}^{+}\right.$) strains compared to $\triangle \mathrm{rraA}$ (AR247 and AR248) after $40 \mathrm{~h}$ at RT. c Diagnostic PCRs confirm the absence of the IraA gene (AN4186) on chromosome II (replaced by Af_riboB) and replacement of the mutant pyroA4 allele at locus AN7725 by the IraA expression cassette and Af_pyroA-i.e. substitution of the $2.6 \mathrm{~kb}$ DNA fragment in AR247 by the $5.8 \mathrm{~kb}$ complementation cassette in transformants C1-C4

A. nidulans Ira gene expression is induced on L-rhamnose via RhaR and subject to carbon catabolite repression independently of CreA

Biochemical (LraA activity) and genetic (RT-sqPCR) analyses have suggested that $\operatorname{lraA}$ co-regulates with rhaA and rhaE (see Fig. 3a, b). Two modes of control of the expression of the $A$. nidulans $\alpha$-L-rhamnosidase genes are known: specific induction in the presence of L-rhamnose which requires the function of the zinc binuclear cluster protein RhaR [12, 26], and carbon catabolite repression which involves an as yet undefined regulatory circuit different to that of the wide domain $\mathrm{Cys}_{2} \mathrm{His}_{2}$ 
repressor CreA [12]. We therefore sought to establish whether expression of the genes of the L-rhamnose catabolic pathway (LRA) is similarly controlled. Using appropriately designed primer pairs (Additional file 2: Table S1 and "Materials and methods") RT-qPCR was undertaken to assess the relative abundances of transcripts of the lraA/AN4186, lraB/AN3740 and $\operatorname{lraC} / \mathrm{AN} 5672$ genes, along with those of $r h a A$ and $r h a E$, in total RNAs isolated from mycelia of three genetic backgrounds (wild type, $\Delta r h a R$ and $c r e A^{d} 30$ ) that were initially grown for $18 \mathrm{~h}$ on $0.1 \%$ fructose minimal medium (MM) and subsequently transferred for $3 \mathrm{~h}$ to $\mathrm{MM}$ supplemented with single or mixed carbon sources ( $1 \% \mathrm{~L}$-rhamnose, $1 \% \mathrm{~L}$-lactose, and a $1 \%$ L-rhamnose $+1 \%$ glucose mixture).

Gene induction was examined by assessing the relative abundances of each of the transcripts in the wild type strain after $3 \mathrm{~h}$ in the presence of L-rhamnose compared to lactose. Box and whisker plots presented in Fig. 5a show that like rhaA and $r h a E$, transcript abundance of the three lra genes is greater on L-rhamnose, with $\operatorname{lraA}$ and $l \mathrm{raC}$ being induced approximately 80 - and 35 -fold, respectively; $\operatorname{lr} a B$ transcript abundance also increased but only by a factor of about 6 . Consistent with qualitative observations made previously by northern blotting [12], rhaA and rhaE are strongly induced on L-rhamnose yielding transcript abundances that are 3 orders of magnitude (i.e. $>1000$-fold) greater than that on lactose. Expression of the $\operatorname{lra} A$, $\operatorname{lra} B$ and $\operatorname{lra} C$ genes is thus a consequence of specific induction in the presence of $\mathrm{L}$-rhamnose and not derepression, with $\operatorname{lraA}$ and $\operatorname{lraC}$ being more strongly induced than $\mathrm{lraB}$. Expression analysis in $A$. niger also indicated a lower level of induction of $\operatorname{lraB}$ [22].

To investigate gene induction mediated by the transcriptional activator RhaR, transcript abundancies were examined in isogenic rhaR ${ }^{+}$and rhaR-deleted $(\Delta r h a R)$ mycelia (strains AR271 and AR225, respectively) after their transfer to media containing L-rhamnose (inducing conditions) as sole carbon source. As can be seen in Fig. $5 \mathrm{~b}$ very low abundance ratios $(<1 \%)$ were found for IraA, rhaA and $r h a E$ transcripts thus reflecting considerably reduced expression (2-3 orders of magnitude) of the corresponding genes in the deletion strain. Indeed, the
Cp values recorded for them in the deletion strain were around the limits of detection (Cp 30-35). Similarly to observations in $A$. niger [22], lraB is the gene least influenced by RhaR. These observations confirm the role of RhaR in mediating induction of the LRA pathway in $A$. nidulans.

Our data have indicated that the physiological inducer of rhamnosidase synthesis is a product of the LRA pathway (Fig. 3c) rather than L-rhamnose itself. CCR of LRA reactions preceding production of the inducer would thus lead to a reduced concentration or absence of the latter and therefore a negative effect on target gene induction. To investigate the possible influence of CCR and CreA on the pathway, RT-qPCR was carried out on RNAs isolated from mycelia of the $A$. nidulans wild type $\mathrm{creA}^{+}$(AR5) and the strongly derepressed $c r e A^{d} 30$ mutant (AR305) after transfer to inducing (L-rhamnose) and inducing/ repressing (L-rhamnose + glucose) conditions. As can been seen from the plots in Fig. $5 \mathrm{c}$ expression of the $\operatorname{lra} A, \operatorname{lraB}$ and $\operatorname{lraC}$ genes in the $\mathrm{creA}^{+}$strain is repressed in inducing/repressing medium and this is effected in a manner independent of CreA since this repression is also seen in the $c r e A^{d} 30$ genetic background. Indeed, the degree of glucose repression of each of the genes is similar in both the wild type and $c r e A^{d} 30$ strains. Additionally, the RT-qPCR results are in agreement with our earlier northern blot data for rhaA and rhaE [12].

Taken together, the above RT-qPCR data show that both the induction and repression profiles of the three LRA pathway genes analysed closely parallel those of the rhamnosidase genes rhaA and rhaE. To the best of our knowledge this is the first demonstration of CreA-independent CCR operating on a plant cell wall sugar catabolic pathway in fungi.

Finally, it is also noteworthy that under inducing (1\% L-rhamnose) conditions transcript abundancies of the three catabolic genes were found to be somewhat elevated in the derepressed $\mathrm{creA}^{d} 30$ strain compared to the wild type (Fig. 5d), an effect observed earlier in northern blot analyses of rhaA and rhaE [12]. This indicates a degree of repression by CreA on each of the five genes under inducing conditions.

\footnotetext{
(See figure on next page.)

Fig. 5 Analysis of Ira and rha gene expression. Relative abundances of Ira and $r$ ha transcripts: a in wild type mycelia (AR5) $3 \mathrm{~h}$ after transfer to MM containing L-rhamnose as sole carbon source compared to transfer to MM containing lactose; $\mathbf{b}$ in the rhaR-deletion strain (AR225) compared to the $r h a R^{+}$isogenic control (AR271) $3 \mathrm{~h}$ after transfer of each to MM containing L-rhamnose; $\mathbf{c}$ in the isogenic wild type (AR5; $\mathrm{CreA}^{+}$- unshaded box) and creAd 30 (AR305; shaded box) strains $3 \mathrm{~h}$ after transfer to inducing/repressing conditions compared to the corresponding gene and strain $3 \mathrm{~h}$ after transfer to inducing conditions; $\mathbf{d}$ in $\mathrm{creA}^{d} 30$ compared to wild type $3 \mathrm{~h}$ after transfer of each to L-rhamnose-containing medium. Box and whisker plots were generated using the program REST-2009 (Qiagen). The box areas of the plots encompass 50\% of all observations, the dotted line represents the sample median and each whisker represents the outer $25 \%$ of observations. The Cp data used in these analyses were obtained from three independent biological replicates and RT-qPCR was done using three technical replicates. The genes encoding histone H2B (AN3469) and beta-tubulin benA (AN1 182) were used as references for normalisation of expression. Primer pairs used were: IraA 398/399; IraB 404/405; IraC 346/347; rhaA 410/411; rhaE 352/353; H2B 394/395; benA 386/387
} 


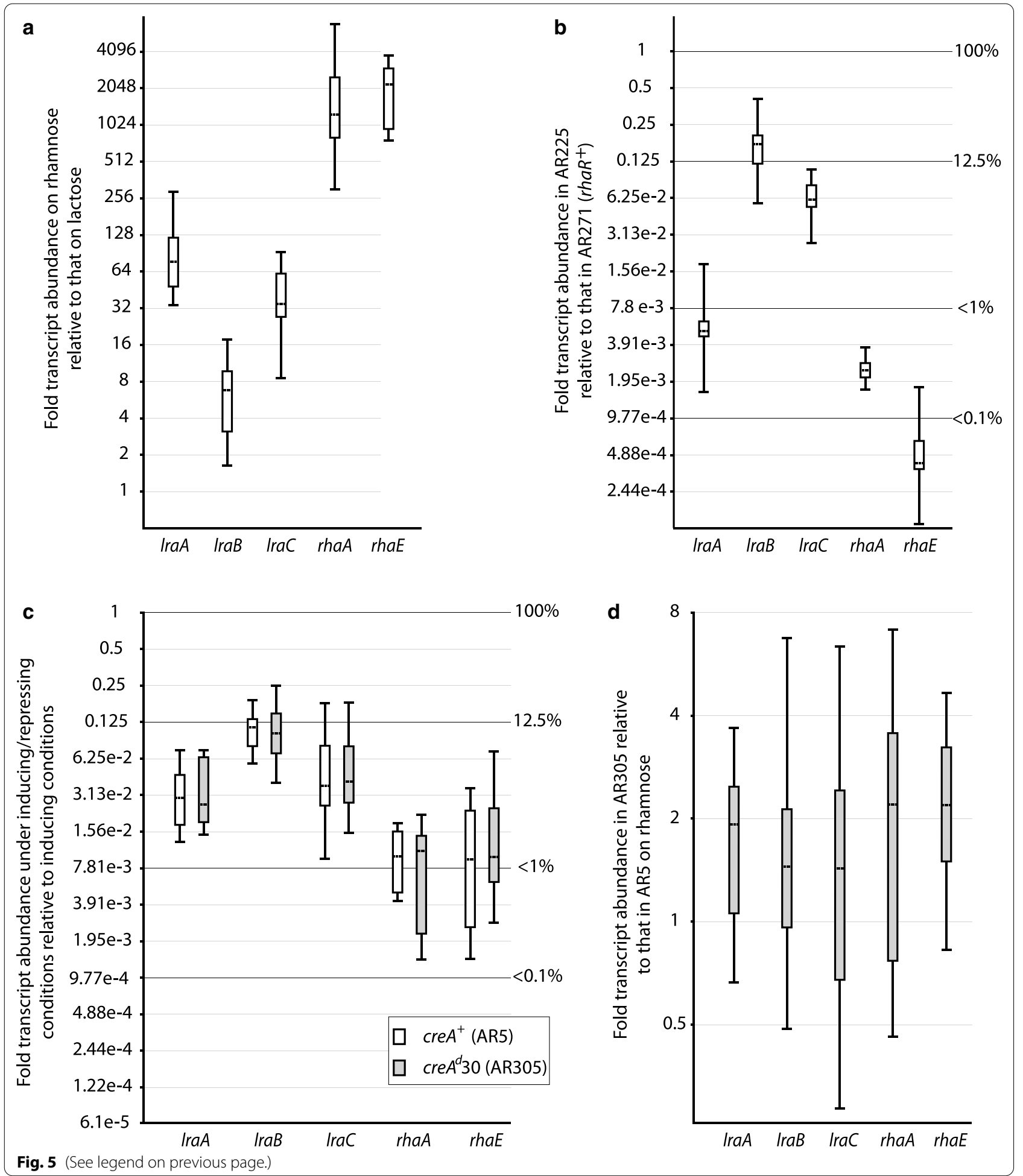

Deletion of IraA/AN4186 negatively affects the expression of L-rhamnose-inducible genes

To corroborate the influence of the LRA pathway on the expression of those genes induced in the presence of L-rhamnose via RhaR we examined transcript abundances in the $\triangle l r a A$ mutant (AR247) relative to those of the isogenic riboflavin nutritional control AR271 $\left(\operatorname{lraA} A^{+}\right)$under inducing conditions. As can be seen 


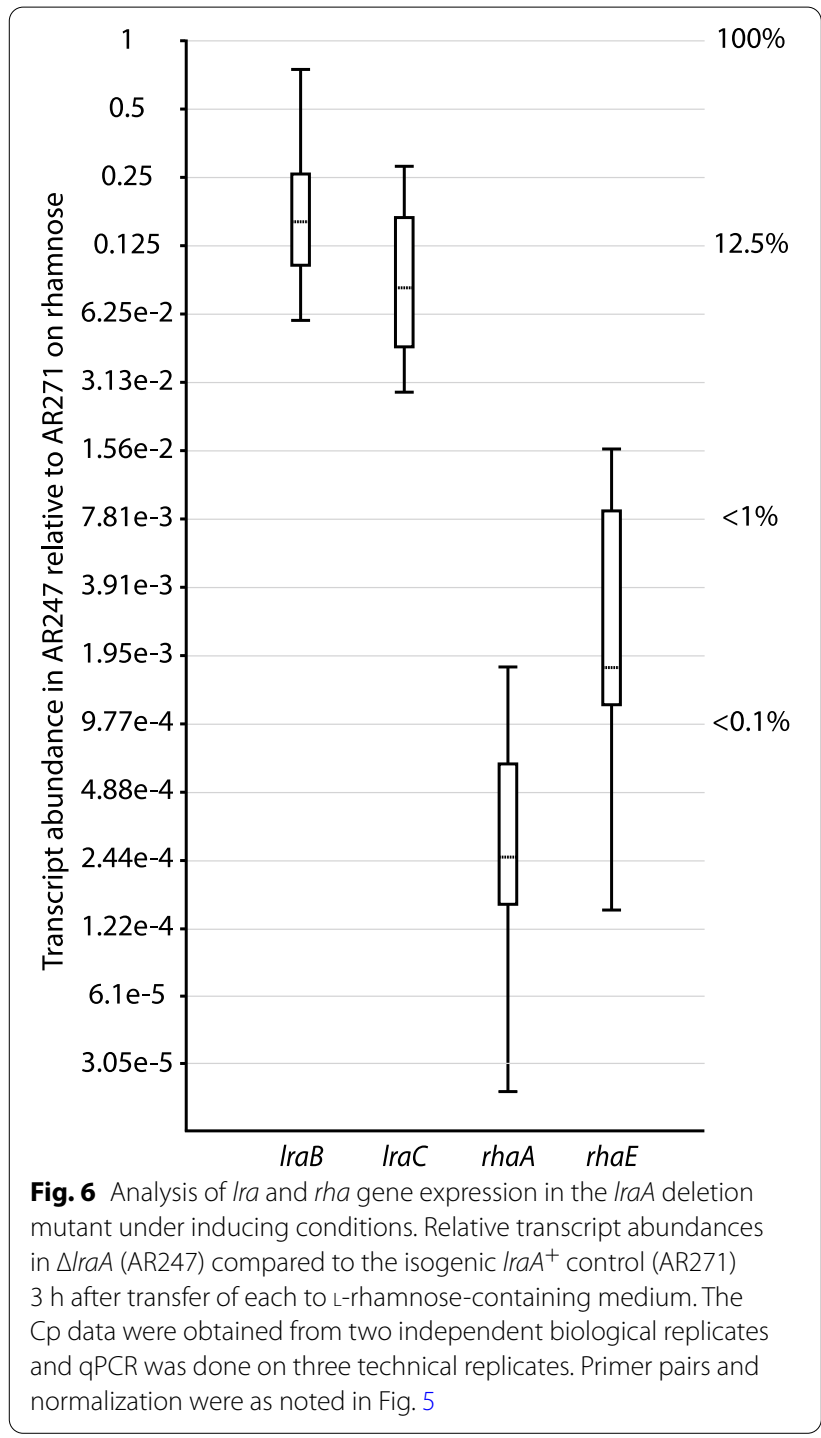

in Fig. 6, relative transcript levels of the genes of the catabolic pathway as well as those encoding the rhamnosidases were reduced in the absence of a functional pathway-in a manner approximately inversely proportional to the RhaR-mediated induction of each on L-rhamnose-thus showing the importance of the pathway for induction.

\section{Conclusions}

In summary, $A$. nidulans genes ( $\operatorname{ra} A, \operatorname{lra} B$ and $\operatorname{lraC}$ ) that are candidates for encoding the first three L-rhamnose catabolic activities of the phosphorylated intermediate (LRA) pathway have been identified by virtue of sequence homologies shared with the products of previously characterized $L R A$ genes in S. stipitis [11, 20]. The phenotype of $\mathrm{lraA} / \mathrm{AN} 4186$ deletion mutants has demonstrated the importance of the LRA pathway for both the utilization of L-rhamnose and the induction of rhamnosidase genes. Data from expression analyses are consistent with the involvement of the $\operatorname{lraB}$ and lra $C$ genes, as well as $\operatorname{lraA}$, in the $\mathrm{L}$-rhamnose catabolic pathway, and have also revealed the novelty that CCR of the LRA pathway is mediated by an as yet unknown non-CreA mechanism.

\section{Supplementary information}

Supplementary information accompanies this paper at https://doi.org/10. 1186/s12934-020-01443-9.

Additional file 1: Figure S1. Schemes of microbial L-rhamnose catabolic pathways. (A) Catabolic pathways for L-rhamnose and (B) organisation of the genes encoding the corresponding activities in $E$. coli and various fungi. Homologous genes are indicated using the same colour code. Chr: chromosome number.

Additional file 2: Table S1. Oligonucleotide primers used in this study.

\section{Acknowledgements}

E.N. was a recipient of an Erasmus Placement fellowship. We wish to thank the recipients of Erasmus fellowships Irene Bardani and Eleftheria Klagou for their contribution to the RT-qPCR data, and the Central Service for Experimental Research (SCSIE) of the University of Valencia for DNA sequencing and RNA-Seq analysis. We acknowledge support of the publication fee by the CSIC Open Access Publication Support Initiative through its Unit of Information Resources for Research (URICI).

\section{Authors' contributions}

$\mathrm{MO}$ and APM coordinated the work undertaken. All authors participated in carrying out experiments and interpreting results. MO and APM wrote the manuscript. All authors read and approved the final manuscript.

\section{Funding}

This work was supported by the Spanish Ministerio de Ciencia e Innovación/ FEDER and Ministerio de Economía y Competitividad/FEDER (Grant Numbers AGL2011-29925 and AGL2015-66131-C2-2-R, respectively.

\section{Availability of data and materials}

All data generated or analysed during this study are included in this published article and its additional files. The A. nidulans cDNA sequence of IraA has been deposited with GenBank (Accession number: MT431619).

\section{Ethics approval and consent to participate}

Not applicable.

\section{Consent for publication \\ Not applicable.}

\section{Competing interests}

The authors declare that they have no competing interests.

\section{Author details}

${ }^{1}$ Instituto de Agroquímica y Tecnología de Alimentos (IATA), Consejo Superior de Investigaciones Científicas (CSIC), c/Catedrático Agustín Escardino Benlloch 7, 46980 Paterna, Valencia, Spain. ${ }^{2}$ Present Address: Center for Basic Research, Biomedical Research Foundation, Academy of Athens (BRFAA), 4 Soranou Ephessiou Street, 11527 Athens, Greece. ${ }^{3}$ Present Address: ADM Biopolis, Parque Científico Universidad de Valencia, c/Catedrático Agustín Escardino Benlloch 9, 46980 Paterna, Valencia, Spain.

Received: 22 April 2020 Accepted: 25 September 2020

Published online: 02 October 2020 


\section{References}

1. Mistou MY, Sutcliffe IC, van Sorge NM. Bacterial glycobiology: rhamnosecontaining cell wall polysaccharides in Gram-positive bacteria. FEMS Microbiol Rev. 2016;40:464-79.

2. Malawista I, Davidson EA. Isolation and identification of rhamnose from rabbit skin. Nature. 1961;192:871-2.

3. Mohnen D. Pectin structure and biosynthesis. Curr Opin Plant Biol. 2008;11:266-77.

4. Harholt J, Suttangkakul A, Scheller HV. Biosynthesis of pectin. Plant Physiol. 2010;153:384-95.

5. Bonnin E, Garnier C, Ralet MC. Pectin-modifying enzymes and pectinderived materials: applications and impacts. Appl Microbiol Biotechnol. 2014;98:519-32.

6. Lahaye M, Robic A. Structure and functional properties of ulvan, a polysaccharide from green seaweeds. Biomacromol. 2007;8:1765-74.

7. Chiellini F, Morelli A. Ulvan: A versatile platform of biomaterials from renewable resources. In: Pignatello R, editor. Biomaterials-physics and chemistry. London: InTech; 2011. p. 75-98.

8. Manzanares P, Vallés S, Ramón D, Orejas M. a-I-Rhamnosidases: old and new insights. In: Polaina J, MacCabe AP, editors. Industrial enzymes: structure, function and applications. Dordrecht: Springer; 2007. p. 117-140.

9. Rodionova IA, Li X, Thiel V, Stolyar S, Stanton K, Fredrickson JK, Bryant DA Osterman AL, Best AA, Rodionov DA. Comparative genomics and functional analysis of rhamnose catabolic pathways and regulons in bacteria. Front Microbiol. 2013;4:407.

10. Koivistoinen OM, Arvas M, Headman MJR, Andberg M, Penttilä M, Jeffries TW, Richard P. Characterization of the gene cluster for L-rhamnose catabolism in the yeast Scheffersomyces (Pichia) stipitis. Gene. 2012;492:177-85.

11. Watanabe S, Saimura M, Makino K. Eukaryotic and bacterial gene clusters related to an alternative pathway of nonphosphorylated L-rhamnose metabolism. J Biol Chem. 2008;283:20372-82.

12. Tamayo-Ramos JA, Flipphi M, Pardo E, Manzanares P, Orejas M. L-Rhamnose induction of Aspergillus nidulansa-L-rhamnosidase genes is glucose repressed via a CreA-independent mechanism acting at the level of inducer uptake. Microbial Cell Fact. 2012;11:26.

13. Sloothaak J, Odoni DI, Martins dos Santos VAP, Schaap PJ, Tamayo-Ramos JA. Identification of a novel L-rhamnose uptake transporter in the filamentous fungus Aspergillus niger. PLoS Genet. 2016;12:e1006468.

14. Badía J, Baldomà L, Aguilar J, Boronat A. Identification of the rhaA, rhaB and rhaD gene products from Escherichia coli K-12. FEMS Microbiol Lett. 1989;53:253-7

15. Moralejo P, Egan SM, Hidalgo E, Aguilar J. Sequencing and characterization of a gene cluster encoding the enzymes for L-rhamnose metabolism in Escherichia coli. J Bacteriol. 1993;175:5585-94.

16. Rigo LU, Makano M, Veiga IA, Feingold DS. L-Rhamnose dehydrogenase of Pullularia pullulans. Biochim Biophys Acta. 1976;445:286-93.

17. Rigo LU, Marechal LR, Vieira MM, Veiga LA. Oxidative pathway for L-rhamnose degradation in Pullularia pullulans. Can J Microbiol. 1985;31:817-22.

18. Twerdochlib AL, Pedrosa FO, Funayama S, Rigo LU. L-Rhamnose metabolism in Pichia stipitis and Debaryomyces polymorphus. Can J Microbiol. 1994:40:896-902.

19. Watanabe S, Makino K. Novel modified version of nonphosphorylated sugar metabolism - an alternative L-rhamnose pathway of Sphingomonas sp. FEBS J. 2009;276:1554-677.

20. Koivistoinen OM, Hilditch S, Voutilainen SP, Boer H, Penttilä M, Richard P. Identification in the yeast Pichia stipitis of the first L-rhamnose-1-dehydrogenase gene. FEBS J. 2008;275:2482-8.

21. Liu B, Zhang Y, Zhang X, Yan C, Zhang Y, Xu X, Zhang W. Discovery of a rhamnose utilization pathway and rhamnose-inducible promoters in Pichia pastoris. Scientific Reports. 2016:6:27352.

22. Khosravi C, Kun KS, Visser J, Aguilar-Pontes MV, de Vries RP, Battaglia E. In vivo functional analysis of L-rhamnose metabolic pathway in Aspergillus niger: a tool to identify the potential inducer of RhaR. BMC Microbiol. 2017;17:214.
23. Vieira MM, Rigo LU, Maréchal LR, Veiga LA. Induction and catabolite repression of L-rhamnose dehydrogenase in Pullularia pullulans. J Bacteriol. 1979:38:55-9.

24. Pardo EH, Funayama S, Pedrosa FO, Rigo LU. Pichia stipitisL-rhamnose dehydrogenase and a catabolite-resistant mutant able to utilize 2-deoxyD-glucose. Can J Microbiol. 1992;38:417-22.

25. Orejas M, Ibáñez E, Ramón D. The filamentous fungus Aspergillus nidulans produces an a-L-rhamnosidase of potential oenological interest. Lett Appl Microbiol. 1999:28:383-8.

26. Pardo E, Orejas M. The Aspergillus nidulans $\mathrm{Zn}(\mathrm{II})_{2} \mathrm{Cys}_{6}$ transcription factor AN5673/RhaR mediates L-rhamnose utilization and the production of a-ı-rhamnosidases. Microb Cell Fact. 2014;13:161.

27. Nayak T, Szewczyk E, Oakley CE, Osmani A, Ukil L, Murray SL, Hynes MJ, Osmani SA, Oakley BR. A versatile and efficient gene-targeting system for Aspergillus nidulans. Genetics. 2006;172:1557-666.

28. Pontecorvo G, Roper JA, Hemmons LM, Macdonald KD, Bufton AWJ. The genetics of Aspergillus nidulans. Adv Genet. 1953;5:141-238.

29. Cove DJ. The induction and repression of nitrate reductase in the fungus Aspergillus nidulans. Biochim Biophys Acta. 1966;113:51-6.

30. Tilburn J, Scazzocchio C, Taylor GG, Zabicky-Zissman JH, Lockington RA, Davies RW. Transformation by integration in Aspergillus nidulans. Gene. 1983;26:205-21.

31. Szewczyk E, Nayak T, Oakley CE, Edgerton H, Xiong Y, Taheri-Talesh N, Osmani SA, Oakley BR. Fusion PCR and gene targeting in Aspergillus nidulans. Nature Protoc. 2007:1:3111-200.

32. Sambrook J, Russell DW. Molecular cloning: a laboratory manual. Cold Spring Harbor: Cold Spring Harbor Laboratory Press; 2001.

33. Martin M. Cutadapt removes adapter sequences from high-throughput sequencing reads. EMBnet J. 2011:17(1):10-2.

34. Kim D, Pertea G, Trapnell C, Pimentel H, Kelley R, Salzberg SL. TopHat2: accurate alignment of transcriptomes in the presence of insertions, deletions and gene fusions. Genome Biol. 2013;14:R36.

35. Varet $H$, Brillet-Guéguen L, Coppee J-Y, Dillies M-A. SARTools: A DESeq2and EdgeR-Based R pipeline for comprehensive differential analysis of RNA-Seq Data. PLoS ONE. 2016;11(6):e0157022.

36. Love MI, Huber W, Anders S. Moderated estimation of fold change and dispersion for RNA-seq data with DESeq2. Genome Biol. 2014;15:550.

37. Vandesompele J, de Preter K, Pattyn F, Poppe B, van Roy N, de Paepe A, Speleman F. Accurate normalization of real-time quantitative RT-PCR data by geometric averaging of multiple internal control genes. Genome Biol. 2002;3(7):research0034.1-research0034.11.

38. Pfaffl MW, Horgan GW, Dempfle L. Relative expression software tool (RESTC) for group-wise comparison and statistical analysis of relative expression results in real-time PCR. Nucl Acids Res. 2002;30:e36.

39. Manzanares $P$, Orejas M, Ibáñez E, Vallés S, Ramón D. Purification and characterization of an a-L-rhamnosidase from Aspergillus nidulans. Lett Appl Microbiol. 2000:31:198-202.

40. Bradford MM. A rapid and sensitive method for the quantitation of microgram quantities of protein utilizing the principle of protein-dye binding. Anal Biochem. 1976;72:248-54.

41. Gruben BS, Zhou M, Wiebenga A, Ballering J, Overkamp KM, Punt PJ, de Vries RP. Aspergillus niger RhaR, a regulator involved in L-rhamnose release and catabolism. Appl Microbiol Biotechnol. 2014:98:5531-40.

42. Thieme N, Wu WW, Dietschmann A, Salamov AA, Wang M, Johnson J, Singan VR, Grigoriev IV, Glass NL, Somerville CR, Benz JP. The transcription factor PDR-1 is a multi-functional regulator and key component of pectin deconstruction and catabolism in Neurospora crassa. Biotechnol Biofuels. 2017;10:149.

43. Mackay EM, Pateman JA. The regulation of urease activity in Aspergillus nidulans. Biochem Genet. 1982;20:763-76.

\section{Publisher's Note}

Springer Nature remains neutral with regard to jurisdictional claims in published maps and institutional affiliations. 